\title{
SUR LA BIOLOGIE DU CASTOR DU RHÔNE (CASTOR FIBER L.)
}

\author{
PAR \\ ACHILLE URBAIN \\ Professeur au Muséum National d'Histoire Naturelle, Paris
}

Le Castor du Rhône (Castor fiber L.) a été particulièrement bien étudié par de nombreux auteurs. Victor Piraud ${ }^{1}$ ) a donné d'intéressants renseignements sur ses moeurs; M. P. Cordier ${ }^{2}$ ) et A. Hugues ${ }^{3}$ ) ont fait aussi une étude appronfondie de cet animal et L. Germand ${ }^{4}$ ) a fourni une description détaillée des colonies de Castors de la région Avignonaise:

Autrefois très abondant en France où il habitait la plupart des rivières, le Castor n'existe plus actuellement que sur le Rhône, certains de ses affluents et en Camargue. Grâce aux mesures énergiques de protection dont il est l'objet depuis un certain nombre d'années, il s'est multiplié et actuellement le nombre de ces colonies ne fait que s'accroître. Les stations les plus réputées sont les suivantes :

Les îles de l'embouchure de la Cèze: îles des Rats, du Colombier, de la Piboulette,

L'île Pilet Ranchier,

Le petit Rhône,

Le pont du Gard

Le parc de Camargue.

Vingt kilomètres du Rhône étaient déclarés réserve protégée, comprenant les estuaires de l'Aigues, de la Cèze et le Canal de Roquemaure, les îles du Colombier, des Rats, de la Piboulette et de Cadolet.

Les colonies de Castors sont en 1949 bien connues. Le nombre des Castors a augmenté d'une façon sensible. On rencontre ces animaux en abondance dans la Vallée du Gardon, outre le Pont du Gard, ils se trouvent à Collias, dans le

I) V. Piraud, Le Castor du Rhône ( $3^{\circ}$ Congrès du Rhône), Genève, 1939, p. 218.

2) M. P. Cordier, La Protection du Castor du Rhône. Bull. Soc. Nat. Acclimatation, I932, p.p. I et 50.

3) A. Hugues, Le Castor du Rhône. Bull. Soc. Nat. Acclimatation, 1933, p.p. 34I, 38I et 425 .

4) L. Germand, Contribution à l'étude des Castors de la Région Avignonaise. Bull. Soc. Etude des Sciences Naturelles du Vaucluse, 1939, $n^{\circ} 2$ et 3. 
Canyon du Gardon, à St Geniès de Malgoirès et dans différents autres points de la Vallée. Des colonies existent même à proximité des Villes, c'est ainsi qu'on en a situé à l'île de la Barthelasse, près d'Avignon. La carte ci-jointe (fig. I) donnera une idée assez exacte de la répartition du Castor dans la Vallée du Rhône.

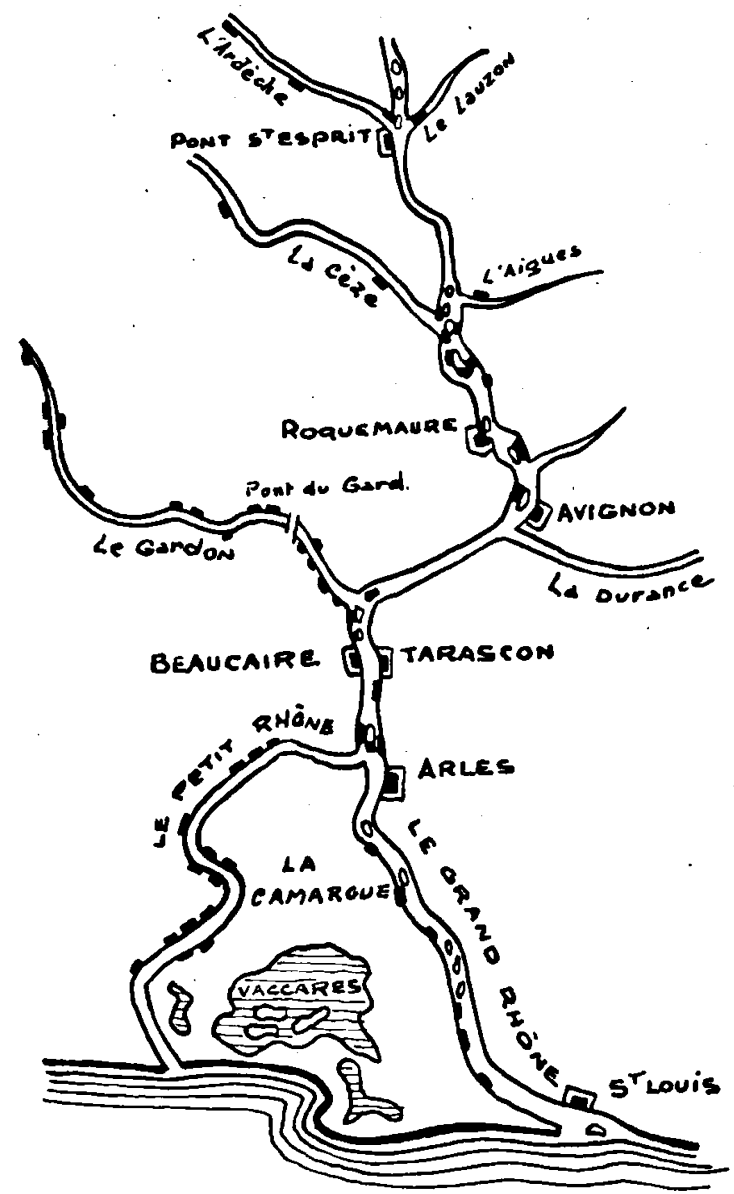

Fig. I. Répartition géographique du Castor en France (d'après Cordier). Les colonies de Castor sont indiquées par des taches noires le long du fleuve et de ses affluents.

Le Castor du Rhône est un animal tranquille, il a abandonné ses qualités d'architecte, son instinct de construction qui l'incitait à faire des huttes et à établir des digues pour rehausser le niveau des rivières, pour devenir un animal fouisseur, creusant des terriers sur les berges du fleuve. Cet animal qui a été pourchassé d'une façon systématique aurait depuis longtemps disparu définitivement s'il avait continué à construire des huttes facilement repérables par 
l'homme. Il vit actuellement dans un terrier qui, d'après CoRDIER, affecte la forme suivante: il prend naissance sur la rive, à soixante centimètres environ sous l'eau, il pénètre dans la berge par un tunnel en pente douce et sur une longueur de I $\mathrm{m}$. à I $\mathrm{m}$. 50, il s'élève assez brusquement selon la hauteur de la berge et affecte la forme d'un couloir qui aboutit à une sorte de rotonde. C'est la chambre, le lieu de repos de la famille. De cette rotonde part un coulour qui, long souvent de plusieurs mètres, vient rejoindre le sol de la berge élevée. Cette sortie, c'est la cheminée. Souvent, il y a un second embranchement sans issue.

Ce terrier classique a une fonction essentielle mais temporaire, il sert surtout à abriter les nouveaux-nés. Comme chaque inondation peut le couvrir, l'embourber ou le détruire, il ne reprend sa forme qu'au printemps, c'est-à-dire au moment où la femelle va mettre bas. $\mathrm{Si}$ après cette période une crue l'a obstrué, il reste toute l'année sans modification. Environ trois semaines avant la gestation, on distingue alors nettement à nouveau la fréquentation de l'entrée par la blancheur du sable, le déblai et l'apport de branches vertes récemment coupées. En effet, lorsqu'il travaille à la réfection de la demeure qui doit abriter sa progéniture, le Castor apporte et cherche à enfermer avec lui une provision de nourriture quotidienne. Ce terrier est soigneusement établi: la propreté y règne: la construction de ce que l'on peut appeler la chambre est une merveille. Cette chambre est ronde, le sol sablonneux est parfaitement horizontal. Comme les racines d'arbres traversent la terre, on constate qu'elles sont toutes coupées au ras de la paroi, comme au rasoir. Les griffes des pattes antérieures servent à faire tomber la terre qui dépasserait l'arrondi voulu et projeté. Dents et griffes servent à façonner une oeuvre d'art.

Le dôme de la chambre a une forme concave; d'ordinaire, il a o m. 70 de diamètre, sur $0 \mathrm{~m}$. 60 de hauteur. On ne saurait mieux le comparer qu'à un grand chaudron renversé.

Lorsque l'entrée est parfaitement aménagée, le couloir bien tracé, avec, dès la sortie de l'eau, une petite plate-forme qui permet au Castor de s'arrêter le temps nécessaire pour sécher ses jarres, il s'occupe alors de l'aération de son habitat. La cheminée est creusée; elle arrive au sol par un couloir assez étroit, souvent sinueux; il semble que le Castor craigne l'air. Une habitude qui ne se dément jamais est d'obstruer cette entrée par des bois coupés et toujours rongés. Ils ne sont pas rangés sans art; taillés en biseau, la pointe du bâton est dirigée vers la sortie; ils sont souvent entre-croisés; véritables chevaux de frise, ils menacent les intrus qui voudraient s'introduire par le haut.

Nous donnons, d'autre part, le plan et la coupe d'un terrier de Castor existant actuellement dans l'île de la Barthelasse, près d'Avignon (fig. 2).

Les essences que goûtent plus particulièrement les Castors du Rhône, sont le Saule, puis le Peuplier blanc. L'Aulne, l'Orme et le Frêne sont aussi appréciés. Ils débitent les branches des arbres abattus, en morceaux d'une longueur d'un mètre et il les entraîne aux abords de son terrier. Il faut signaler que si 
ce rongeur est susceptible de s'attaquer à de grands arbres, il préfère le moindre effort et exploite de préférence les arbrisseaux de faible taille. Il utilise aussi avec empressement les branches abandonnées par les bucherons.

Les Castors, lorsqu'ils ont choisi un arbre qui convient à leur goût, l'abattent sans laisser aucune trace, aucune brindille ni feuille ne sont trouvées sur place. Quelques copeaux seuls par leur présence au pied des saules abattus, attestent l'activité de ces rongeurs.

Les lieux d'abattage d'arbres situés en pleine broussaille sont reliés à la rive par des passages taillés par les incisives des Castors et forment de véritables

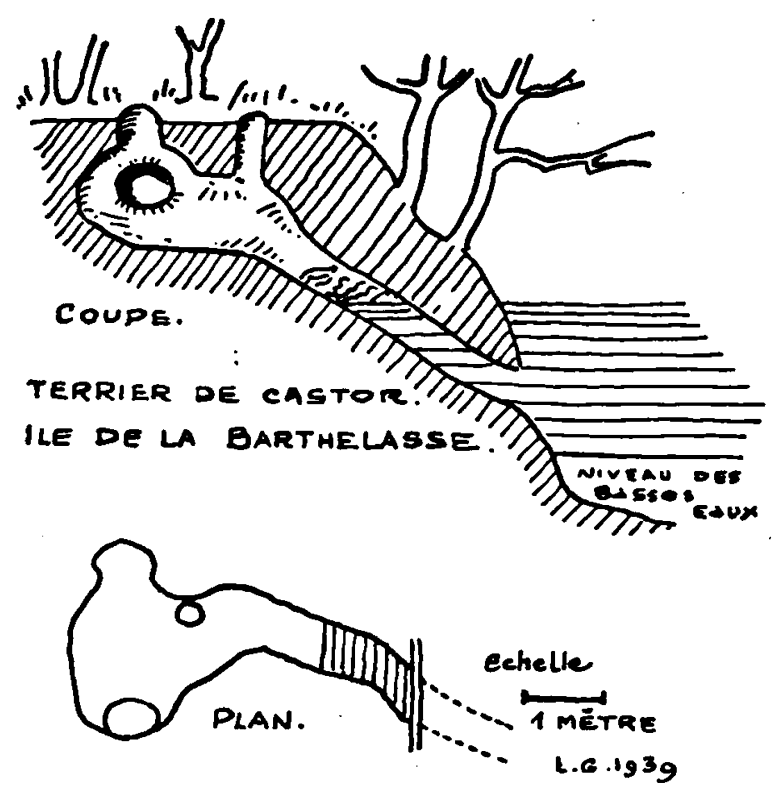

Fig. 2. Plan et coupe d'un terrier de la Barthelasse (d'après L. Germand).

tunnels dans la végétation; on distingue sur le sol de ces tunnels la trace des charrois. Comme ces sentiers descendent vers l'eau, ils sont bien visibles du milieu de la rivière.

Le Castor du Rhône n'est par ordinairement polygame. Dans la nature ces animaux vivent le plus souvent par couples. La femelle donne de un à six petits. Le jeune Castor ne voit qu'au bout de huit jours. C'est une petite boule de fourrure qui se déplace difficilement et qui ne sait pas nager. C'est la mère qui en prend soin, qui le guide dans ses premiers ébats dans l'eau, qui lui apprend peu à peu à plonger... A trois mois il est encore maladroit. Jeune, il reste peu de temps sous l'eau; il lui faut de l'entrainement pour rester plusieurs minutes sans respirer.

Les Castors de deux ans quittent leurs parents pour fonder des colonies nouvelles. 
Quand il a épuisé la nourriture qui lui convient dans une région, le Castor émigre vers d'autres lieux où il est sûr de trouver sa subsistance. Les migrations ne sont déterminées que par la faim.

Les deux seuls ennemis du Castor du Rhône sont la Loutre et les crues du fleuve qui détruisent les terriers et noient les jeunes:

$\mathrm{Ce}$ rongeur s'apprivoise facilement, il vit bien en captivité où parfois il devient familier ${ }^{5}$ ).

5) Voir à ce sujet Paul Corvier-Goni, Castors du Rhône. Ed. Albin Michel, Paris, I乌̧7. 